\title{
Piezoelectric surface acoustical phonon limited mobility of electrons in graphene on a GaAs substrate
}

\author{
S. H. Zhang, ${ }^{1,2}$ W. Xu, ${ }^{2}$ S. M. Badalyan,,${ }^{1, *}$ and F. M. Peeters ${ }^{1}$ \\ ${ }^{1}$ Department of Physics, University of Antwerp, Groenenborgerlaan 171, B-2020 Antwerpen, Belgium \\ ${ }^{2}$ Key Laboratory of Materials Physics, Institute of Solid State Physics, Chinese Academy of Sciences, Hefei 230031, China
}

(Received 28 November 2012; published 26 February 2013)

\begin{abstract}
We study the mobility of Dirac fermions in monolayer graphene on a GaAs substrate, limited by the combined action of the extrinsic potential of piezoelectric surface acoustical phonons of GaAs (PA) and of the intrinsic deformation potential of acoustical phonons in graphene (DA). In the high-temperature ( $T$ ) regime, the momentum relaxation rate exhibits the same linear dependence on $T$ but different dependencies on the carrier density $n$, corresponding to the mobility $\mu \propto 1 / \sqrt{n}$ and $1 / n$, respectively for the PA and DA scattering mechanisms. In the low- $T$ Bloch-Grüneisen regime, the mobility shows the same square-root density dependence $\mu \propto \sqrt{n}$, but different temperature dependencies $\mu \propto T^{-3}$ and $T^{-4}$, respectively for PA and DA phonon scattering.
\end{abstract}

DOI: 10.1103/PhysRevB.87.075443

PACS number(s): 72.80.Vp, 72.10.-d, 73.40.-c, 77.65.Dq

\section{INTRODUCTION}

Graphene, ${ }^{1}$ due to its unique linear chiral electronic dispersion, ${ }^{2}$ exhibits novel transport properties $^{3,4}$ and has great potential as a desirable material for future electronic and optical technologies. ${ }^{2,5}$ Momentum relaxation is a key phenomenon that governs transport of Dirac fermions in graphene. ${ }^{6}$ It is of practical interest for developing high-speed electronics and in recent years has been extensively studied both theoretically ${ }^{7-9}$ and experimentally. ${ }^{10-13}$ The scattering by defects, ${ }^{7,14-17}$ impurities, ${ }^{18-22}$ and phonons ${ }^{9,12,17,23-28}$ has been investigated to determine and control the dominant mechanism that limits the carrier mobility in graphene.

In device structures, graphene is often deposited on a $\mathrm{SiO}_{2}$ wafer, which imposes constraints on the excellent transport properties observed in suspended graphene. ${ }^{10,29}$ Recently, structures on such promising substrate materials as $h$-BN (Refs. 30 and 31) and GaAs (Refs. 32 and 33) have been fabricated and studied with the intention for high-quality graphene electronics. Along with its superior surface quality and strong hydrophilicity preventing folding of large-scale graphene flakes, GaAs has a substantially larger dielectric constant in comparison with $\mathrm{SiO}_{2}$ and $h$-BN hence improved electrical screening. In such high-purity GaAs structures, electron-phonon scattering can be a decisive factor in limiting the mobility of Dirac fermions and the piezoelectric GaAs substrate can serve as a powerful tool for studying the electronic properties of graphene by means of remote surface acoustical phonons.

In this work, we study the temperature and density dependencies of the carrier mobility in monolayer graphene on a GaAs substrate. We calculate the mobility limited by scattering from the piezoelectric potential of surface acoustical phonons of the substrate (PA phonons) versus the deformation potential of acoustical phonons of graphene (DA phonons). In experiment, the typical wavelength of phonons taking part in scattering events is much larger than the distance $d$ of several angströms between the graphene sheet and the GaAs substrate so that the extrinsic interaction of PA phonons with Dirac fermions is quite strong and, as we shall see, can dominate the intrinsic interaction of DA phonons.

\section{THEORETICAL MODEL}

\section{A. Extrinsic PA scattering}

Crystal surfaces modify substantially bulk phonon modes and can change qualitatively the carrier relaxation characteristics. ${ }^{34,35}$ In crystals with lack of a center of symmetry such as in GaAs, the displacement field of Rayleigh waves (a combination of longitudinal and transversal oscillations) propagating on a crystal-free surface ${ }^{36}$ induces a piezoelectric polarization of the lattice. It leads to an electric potential both inside and outside of the GaAs substrate that couples to the electrons in graphene. The Hamiltonian of such piezoelectric interaction between surface acoustical phonons of GaAs and Dirac fermions in graphene can be written as

$$
H_{\mathrm{e}-\mathrm{ph}}^{\mathrm{PA}}=e \varphi(\mathbf{R}, t)=\frac{1}{\sqrt{\mathcal{A}}} \sum_{\mathbf{q}} \gamma_{q}^{\mathrm{PA}} e^{i \mathbf{q R}} b_{\mathbf{q}}+\text { c.c. },
$$

where the electric potential $\varphi(\mathbf{R}, t)$ in the plane of the graphene sheet is given by the solution of the Poisson equation as $\varphi(\mathbf{R}, t) \propto \hat{q}_{x} \hat{q}_{y} e^{i\left(\mathbf{q R}-\omega_{q} t\right)} e^{-q d} .37$ Here, $q$ and $\omega_{q}=v_{\mathrm{PA}} q$ are the phonon momentum and energy with the velocity of Rayleigh waves $v_{\mathrm{PA}} \approx 0.9 v_{b} \approx 2.7 \times 10^{3} \mathrm{~m} / \mathrm{s}, v_{b}$ is the bulk sound velocity in GaAs, and $\hat{q}_{x, y}=q_{x, y} / q$. In Eq. (1), $b_{\mathbf{q}}$ denotes the amplitude of the phonon field and the piezoelectric electron-phonon interaction vertex is defined as $\left|\gamma_{q}^{\mathrm{PA}}\right|^{2}=c_{\mathrm{PA}}^{2}\left(\hat{q}_{x} \hat{q}_{y}\right)^{2} e^{-2 q d} \hbar^{2} v_{\mathrm{PA}} /\left(p_{0} \bar{\tau}_{P A}\right)$ where we use the nominal time $\bar{\tau}_{\mathrm{PA}} \approx 8 \mathrm{ps}$, introduced in Ref. 38 for carrier scattering from bulk piezoelectric acoustical phonons. Here, the characteristic wave vector $p_{0}=2.5 \times 10^{6} \mathrm{~cm}^{-1}$ is related to the optical phonon energy in GaAs and the numerical factor $c_{\mathrm{PA}} \approx 4.9$ is determined by the elastic properties of GaAs. ${ }^{37}$ The strongest electron-PA phonon interaction takes place for surface phonons propagating along the diagonal direction with $q_{x} \approx q_{y}$ so that in (1) we can approximate $\left(\hat{q}_{x} \hat{q}_{y}\right)^{2} \approx \frac{1}{4}$. Taking also $e^{-2 q d} \approx 1$ for typical values of $d \sim 5 \AA,,^{32,33}$ one can see that $\gamma_{q}^{\mathrm{PA}}$ in this well-justified approximation is independent of $q$. This differs from the linear wave-vector dependence of the DA interaction vertex and results in a new, qualitatively different, contribution to the mobility. 


\section{B. Intrinsic DA scattering}

The Hamiltonian of electron-acoustical phonon interaction due to the deformation potential in graphene can be written as

$$
H_{\mathrm{DA}}=\frac{1}{\sqrt{\mathcal{A}}} \sum_{\mathbf{q}} \gamma_{q}^{\mathrm{DA}} e^{i \mathbf{q R}} b_{\mathbf{q}}+\text { c.c. }
$$

with the DA interaction vertex defined as $\left|\gamma_{q}^{\mathrm{DA}}\right|^{2}=$ $\hbar^{2} q v_{\mathrm{DA}} /\left(p_{0}^{2} \bar{\tau}_{\mathrm{DA}}\right)$. Here, we introduce the nominal scattering time $1 / \bar{\tau}_{\mathrm{DA}}=\Xi^{2} p_{0}^{2} /\left(2 \hbar \rho v_{\mathrm{DA}}^{2}\right)$ for DA phonons and find $\bar{\tau}_{\mathrm{DA}} \approx$ $0.8 \mathrm{ps}$ for the following values of the graphene parameters ${ }^{9}$ : $\Xi=6.8 \mathrm{eV}$ the deformation potential constant, $\rho=7.6 \times$ $10^{-7} \mathrm{~kg} / \mathrm{m}^{2}$ the mass density, and $v_{\mathrm{DA}}=2.0 \times 10^{4} \mathrm{~m} / \mathrm{s}$ the sound velocity in graphene, which is essentially larger than the sound velocity in GaAs. The direct comparison $\left|\gamma_{q}^{\mathrm{PA}}\right|^{2} /\left|\gamma_{q}^{\mathrm{DA}}\right|^{2} \approx 2.0 \times 10^{5} \mathrm{~cm}^{-1} / q$ shows both the PA and DA scattering mechanisms can be important in typical experimental situations and their actual contributions to the mobility are determined by the carrier density $n$ and temperature $T$.

\section{MOMENTUM RELAXATION}

\section{A. Relaxation rate of a test electron}

The momentum relaxation rate of an electron due to scattering from the potential of the $s=\mathrm{PA}$, DA phonons is

$$
\frac{1}{\tau_{1}^{s}\left(\varepsilon_{\lambda \mathbf{k}}\right)}=\sum_{\lambda^{\prime}, \mathbf{k}^{\prime}}^{ \pm, \mathbf{q}}\left(1-\cos \theta_{\mathbf{k} \mathbf{k}^{\prime}}\right) W_{\lambda \mathbf{k} \rightarrow \lambda^{\prime} \mathbf{k}^{\prime}}^{ \pm s \mathbf{q}} \frac{1-f\left(\varepsilon_{\lambda^{\prime} \mathbf{k}^{\prime}}\right)}{1-f\left(\varepsilon_{\lambda \mathbf{k}}\right)}
$$

where $\varepsilon_{\lambda \mathbf{k}}=\lambda v_{F} k$ is the electron energy in graphene with the chirality $\lambda$ and the Fermi velocity $v_{F}$. The Fermi function $f\left(\varepsilon_{\lambda \mathbf{k}}\right)$ is determined by the Fermi energy $\varepsilon_{F}$ and the lattice temperature $T$. The transition probability due to the emission $(+)$ and absorption $(-)$ of phonons is

$$
\begin{aligned}
W_{\lambda \mathbf{k} \rightarrow \lambda^{\prime} \mathbf{k}^{\prime}}^{ \pm s \mathbf{q}}= & \frac{2 \pi}{\hbar}\left|M_{\lambda \mathbf{k} \rightarrow \lambda^{\prime} \mathbf{k}^{\prime}}^{ \pm s \mathbf{q}}\right|^{2}\left(N\left(\omega_{s \mathbf{q}}\right)+\frac{1}{2} \pm \frac{1}{2}\right) \\
& \times \delta\left(\varepsilon_{\lambda \mathbf{k}}-\varepsilon_{\lambda^{\prime} \mathbf{k}^{\prime}} \mp \hbar \omega_{s \mathbf{q}}\right)
\end{aligned}
$$

with the Bose factors $N\left(\omega_{s \mathbf{q}}\right)$ representing the number of $s, \mathbf{q}$ phonons. Making use of the electron wave function in graphene $\psi^{T}(\mathbf{R})=\left|e^{-i \theta_{\mathbf{k}}}, \lambda\right| e^{-i \mathbf{k} \cdot \mathbf{R}} / \sqrt{2 \mathcal{A}}$ where $\theta_{\mathbf{k}}$ is the polar angle of the electron wave vector $\mathbf{k}$, we obtain for the square modulus of the PA and DA matrix elements

$$
\left|M_{\lambda \mathbf{k} \rightarrow \lambda^{\prime} \mathbf{k}^{\prime}}^{ \pm s \mathbf{q}}\right|^{2}=\frac{\delta_{\mathbf{k}^{\prime}, \mathbf{k} \mp \mathbf{q}}}{\mathcal{A}}\left|\gamma_{q}^{s}\right|^{2} \mathcal{F}_{\lambda^{\prime} \lambda}\left(\theta_{\mathbf{k k}^{\prime}}\right)
$$

Here, $\theta_{\mathbf{k} \mathbf{k}^{\prime}}=\theta_{\mathbf{k}^{\prime}}-\theta_{\mathbf{k}}$ and the form factor $\mathcal{F}_{\lambda^{\prime} \lambda}\left(\theta_{\mathbf{k} \mathbf{k}^{\prime}}\right)=(1+$ $\left.\lambda \lambda^{\prime} \cos \theta_{\mathbf{k k}^{\prime}}\right) / 2$ represents an overlap of the spinor wave functions. We consider electron-phonon scattering in doped graphene with $\varepsilon_{F}$ much larger than the typical acoustical phonon energy. In such samples, only intrachirality subband transitions are effective so we take $\lambda=\lambda^{\prime}=1$ and omit this index. Then, we represent the momentum relaxation rate due to extrinsic PA and intrinsic DA phonons as

$$
\frac{1}{\tau_{1}^{s}\left(\varepsilon_{\mathbf{k}}\right)}=\frac{a c^{2}}{\pi} \frac{1}{\bar{\tau}_{s}}\left(\frac{k}{p_{0}}\right)^{1+m} \mathcal{G}_{2+m}(x),
$$

where we introduce the function

$$
\mathcal{G}_{k}(x)=\sum_{ \pm} \int_{0}^{z_{ \pm}} d z z^{k} \eta(a, z) \Psi^{ \pm}(x, y)
$$

with $z=q / 2 k$ and $z_{ \pm}=1 /(1 \pm a)$. In Eq. (6) for PA phonons, we take $m=0$ and $a=a_{\mathrm{PA}}=v_{\mathrm{PA}} / v_{F}, c=c_{\mathrm{PA}}$; for DA phonons $m=1, a=a_{\mathrm{DA}}=v_{\mathrm{DA}} / v_{F}, c=c_{\mathrm{DA}}=2 \sqrt{2}$. For brevity, we define $\Psi^{ \pm}(x, y)=\left[N(y)+\frac{1}{2} \pm \frac{1}{2}\right][1-f(x \mp$ $y)] /[1-f(x)]$ where $x=\left(\varepsilon_{\mathbf{k}}-\varepsilon_{F}\right) / T$ and $y=\omega_{s \mathbf{q}} / T=$ $z\left(k / k_{F}\right)\left(T_{\mathrm{BG}}^{s} / T\right)$. The characteristic Bloch-Grüneisen (BG) temperatures are $T_{\mathrm{BG}}^{s}=2 \hbar v_{s} k_{F}$. The function $\eta(a, z)=$ $\sqrt{1-\left[a \pm\left(1-a^{2}\right) z\right]^{2}} /(1 \mp 2 a z)$ restricts backscattering processes due to the chirality. As far as $v_{F} \approx 1.15 \times 10^{6} \mathrm{~m} / \mathrm{s}$ is much larger than $v_{s}$ in GaAs and graphene, in our analytical calculations we take $\eta(a, z) \approx \eta(0, z)=\sqrt{1-z^{2}}$ and $z_{ \pm} \approx 1$.

\section{B. Analytical results in different regimes}

First, we analyze analytically the electron relaxation in the regimes where PA and DA phonon scattering are qualitatively different. For a typical doping level $n=\bar{n} \times 10^{12} \mathrm{~cm}^{-2}$ with $\bar{n} \sim 1$, we have $\varepsilon_{F} \sim 1350 \mathrm{~K}$ and for energies and temperatures up to room temperature, the system of massless Dirac fermions is a well-defined Fermi gas where carrier scattering events due to PA and DA phonons are kinematically quasielastic, i.e., $\left|\varepsilon-\varepsilon_{F}\right|, T \ll \varepsilon_{F}$. Therefore, we consider three typical temperature regions with boundaries given in terms of $T_{\mathrm{BG}}^{s}$. Because $T_{\mathrm{BG}}^{\mathrm{DA}} / T_{\mathrm{BG}}^{\mathrm{PA}} \approx 8$, all three regions are well defined.

\section{High temperatures}

In the high-temperature regime $T \gg T_{\mathrm{BG}}^{s}$, $\mathrm{PA}$ and $\mathrm{DA}$ phonons with energies $\omega_{s \mathbf{q}} \sim T_{\mathrm{BG}}^{s}$ are important in the relaxation processes and because $\omega_{s \mathbf{q}} \ll T$, such scattering events are statically quasielastic. ${ }^{38}$ Under this severe condition, we have $y \ll 1$ so that the Bose factors are large $N(y) \approx 1 / y \gg 1$, and scattering is dominated by induced phonon emission and absorption processes. Therefore, in Eq. (7) to leading order in $y$, we can replace $f(x \pm y)$ by $f(x)$ while $N(y)+\frac{1}{2} \pm \frac{1}{2}$ by $1 / y$. Then, $\mathcal{G}_{2+m}(x) \approx b T / T_{\mathrm{BG}}$ (with $m=0$ and $b=\frac{1}{3}$ for PA and $m=1$ and $b=\pi / 16$ for DA phonons) and for the momentum relaxation rate in this regime, we obtain

$$
\frac{1}{\tau_{1}^{s}\left(\varepsilon_{\mathbf{k}}\right)}=\frac{b c^{2}}{2 \pi} \frac{1}{\bar{\tau}_{s}}\left(\frac{k_{F}}{p_{0}}\right)^{m+1} \frac{T}{T_{F}} .
$$

Here, the relaxation rate for DA phonons reproduces the previous result from Refs. 9. The linear $T$ dependence of $\tau_{1}(\varepsilon)$ both due to PA and DA phonon scattering and its independence on the energy $\varepsilon-\varepsilon_{F}$ are distinctive features of statically quasielastic electron-phonon scattering in the high- $T$ regime where the scale of variation of $\tau_{1}(\varepsilon)$ is $T_{F}$, which is larger than $T_{\mathrm{BG}}^{S}$, and the Pauli exclusion principle does not play an essential role. In this regime, the extrinsic PA and intrinsic DA contributions to $\tau_{1}(\varepsilon)$ differ by a factor

$$
\frac{1 / \tau_{1}^{\mathrm{PA}}\left(\varepsilon_{\mathbf{k}}\right)}{1 / \tau_{1}^{\mathrm{DA}}\left(\varepsilon_{\mathbf{k}}\right)}=\gamma_{H} \frac{p_{0}}{k_{F}} \approx \sqrt{\frac{0.5}{\bar{n}}},
$$


where $\gamma_{H}=\frac{b_{\mathrm{PA}} c_{\mathrm{PA}}^{2} \bar{\tau}_{\mathrm{DA}}}{b_{\mathrm{DA}} c_{\mathrm{DA}}^{2} \overline{\mathrm{T}}_{\mathrm{PA}}} \approx 0.5$ is determined by the elastic properties of graphene and GaAs. Thus, in this regime we find that independent of $\varepsilon-\varepsilon_{F}$ and $T$, the extrinsic PA phonon contribution dominates the intrinsic DA phonon contribution to the momentum relaxation rate at densities smaller than $n=5 \times 10^{11} \mathrm{~cm}^{-2}$ and this enhancement is a square-root function with $n$.

\section{Low temperatures}

In the low-temperature regime $T \ll T_{\mathrm{BG}}^{s}$, if additionally the electron energy is small $\left|\varepsilon-\varepsilon_{F}\right| \ll T_{\mathrm{BG}}^{s}$, electronic transitions are dominated by small-angle scattering events. The typical phonon momenta $q \ll k_{F}$ correspond to the $z \rightarrow 0$ limit and one can extend the integration over $z$ in Eq. (7) up to infinity. Replacing also $1-z^{2}$ by 1 and taking into account that $z \approx y\left(T / T_{\mathrm{BG}}^{s}\right)$, we have $\mathcal{G}_{k}(x) \approx\left(T / T_{\mathrm{BG}}^{s}\right)^{k+1} \mathcal{F}_{k}(x)$ where we define the function $\mathcal{F}_{k}(x)=\sum_{ \pm} \int_{0}^{\infty} d y y^{k} \Psi^{ \pm}(x, y)$. Hence, the momentum relaxation rate in the low- $T$ regime is given as

$$
\frac{1}{\tau_{1}^{s}\left(\varepsilon_{\mathbf{k}}\right)}=\frac{a c^{2}}{\pi} \frac{1}{\bar{\tau}_{s}}\left(\frac{k_{F}}{p_{0}}\right)^{m+1}\left(\frac{T}{T_{\mathrm{BG}}^{s}}\right)^{m+3} \mathcal{F}_{2+m}(x) .
$$

Here, one should distinguish two subregimes. For thermal electrons $\left|\varepsilon-\varepsilon_{F}\right| \lesssim T$, electronic transitions with typical phonon momenta $\hbar q \sim T / v_{s}$ dominate. The function $\mathcal{F}_{k}(x)$ weakly depends on $x$ for $x \lesssim 1$ and can be replaced by $\mathcal{F}_{k}(0)$. Hence, in this $\mathrm{BG}$ regime the relaxation rate (10) exhibits $T^{3}$ and $T^{4}$ dependencies, respectively, for PA and DA phonon scattering. For hot electrons in the opposite $T \rightarrow 0$ limit, transitions are dominated by spontaneous emission of phonons. We have $\mathcal{F}_{k}(x) \approx|x|^{k} /(1+k)$ for $|x| \gg 1$, and the rate (10) does not depend on $T$ and is proportional to $\left(\left|\varepsilon-\varepsilon_{F}\right| / T_{\mathrm{BG}}^{s}\right)^{m+3}$. The direct comparison of the extrinsic PA and intrinsic DA contributions to $\tau_{1}(\varepsilon)$ in the low- $T$ regime gives for thermal electrons

$$
\frac{1 / \tau_{1}^{\mathrm{PA}}\left(\varepsilon_{\mathbf{k}}\right)}{1 / \tau_{1}^{\mathrm{DA}}\left(\varepsilon_{\mathbf{k}}\right)}=\gamma_{L} \frac{p_{0}}{k_{F}} \frac{T_{\mathrm{PA}}}{T},
$$

where the factor $\gamma_{L}=\frac{a_{\mathrm{DA}}^{3} c_{\mathrm{PA}}^{2} \mathcal{F}_{2}(0) \bar{\tau}_{\mathrm{DA}}}{a_{\mathrm{PA}}^{3} c_{\mathrm{DA}}^{2} \mathcal{F}_{3}(0) \bar{\tau}_{\mathrm{PA}}} \approx 41$. For hot electrons, $\gamma_{L}$ is even larger. Thus, in the low- $T$ regime, the intrinsic DA contribution is negligible while the extrinsic PA contribution leads not only to strong enhancement of the momentum relaxation rate but changes its dependence on the lattice temperature from $T^{4}$ to $T^{3}$.

\section{Intermediate temperatures}

In the region of intermediate temperatures $T_{\mathrm{BG}}^{\mathrm{PA}} \ll T \ll$ $T_{\mathrm{BG}}^{\mathrm{DA}}$, small-angle scattering events dominate the DA phonon relaxation with the rate given by Eq. (10), while the PA phonon relaxation is governed by quasielastic large-angle scattering events with the rate (8). Therefore, the relaxation of thermal electrons due to scattering from intrinsic DA phonons is still suppressed in comparison with that from extrinsic PA phonons by a factor

$$
\frac{1 / \tau_{1}^{\mathrm{PA}}\left(\varepsilon_{\mathbf{k}}\right)}{1 / \tau_{1}^{\mathrm{DA}}\left(\varepsilon_{\mathbf{k}}\right)}=\gamma_{I} \frac{p_{0}}{k_{F}}\left(\frac{T_{\mathrm{DA}}}{T}\right)^{3},
$$

where $\gamma_{I}=\frac{b_{\mathrm{PA}} c_{\mathrm{PA}}^{2} \bar{\tau}_{\mathrm{DA}}}{\mathcal{F}_{3}(0) c_{\mathrm{DA}}^{2} \overline{\mathrm{T}}_{\mathrm{PA}}}$. As seen, this suppression depends strongly on $T$, however, due to the small numerical prefactor $\gamma_{I} \approx 4 \times 10^{-3}\left(2.5 \times 10^{-2}\right.$ for hot electrons $)$, it is large only near the low edge $T \gtrsim T_{\mathrm{BG}}^{\mathrm{PA}}$ of this intermediate temperature region with the relaxation rate linear in $T$. Towards the upper edge $T \lesssim T_{\mathrm{BG}}^{\mathrm{DA}}$, the DA phonon contribution to $\tau_{1}(\varepsilon)$ increases and at high densities the PA contribution prevails with the $T^{4}$ behavior of the momentum relaxation rate.

\section{Mobility of Dirac fermions}

Within the Boltzmann transport theory, the electron momentum relaxation time averaged over its energy

$$
\tau_{1}^{s}(n, T)=\int d \varepsilon D(\varepsilon) \tau_{1}^{s}(\varepsilon)[-\partial f(\varepsilon) / \partial \varepsilon],
$$

where $D(\varepsilon)$ is the electron density of states, determines the carrier mobility in graphene as ${ }^{18}$

$$
\mu_{s}(n, T)=\frac{e v_{F}}{\hbar k_{F}} \tau_{1}^{s}(n, T) .
$$

As seen from Eqs. (8) and (10) in the high- $T$ regime as well as for thermal electrons in the low- $T$ BG regime, $\tau_{1}(\varepsilon)$ is independent of $\varepsilon$. Therefore, we find that in the high- $T$ regime the mobility shows the same $\mu \propto T^{-1}$ temperature dependence for both the PA and DA scattering mechanisms, but different density dependencies $\mu \propto 1 / \sqrt{n}$ and $\mu \propto 1 / n$, respectively, for PA and DA phonon scattering. In the low $-T$ BG regime, the mobility exhibits the same density dependence $\mu \propto \sqrt{n}$, but different temperature dependencies $\mu \propto T^{-3}$ and $\mu \propto T^{-4}$, respectively, for PA and DA phonon scattering.

\section{NUMERICAL RESULTS}

In Figs. 1-3, we present our numerical calculations based on Eqs. (6) and (7). In Fig. 1, we study the electron momentum relaxation due to the combined action of extrinsic PA and intrinsic DA phonons. The relaxation rate versus $T$ is plotted for different values of $\varepsilon-\varepsilon_{F}$ and $n$. It is seen in all panels that at low $T$ the rate is almost independent of $T$, which corresponds to the behavior of hot electrons with $\varepsilon-\varepsilon_{F} \gg T$. In the small-angle scattering subregime $T \ll \varepsilon-\varepsilon_{F} \ll T_{\mathrm{BG}}^{s}$, the intrinsic DA scattering is strongly suppressed with respect to the extrinsic PA scattering (cf. the top left and top right panels) and this is consistent with the above discussion that $1 / \tau_{1}^{s} \propto\left(\varepsilon-\varepsilon_{F} / T_{\mathrm{BG}}^{s}\right)^{3+m}$. With an increase of $\varepsilon-\varepsilon_{F}$, this dependence weakens, and in the large-angle scattering subregime $\varepsilon-\varepsilon_{F} \gtrsim T_{\mathrm{BG}}^{s}$, the PA and DA phonon contributions to the relaxation of hot electrons become of the same order and the DA phonon contribution can even dominate at low densities (cf. the bottom right panel). In the high- $T$ regime, as seen in all panels of Fig. 1, the relaxation rate exhibits the same linear $T$ dependence, in agreement with Eq. (8). The PA and DA phonon mechanisms make equal contributions to the rate at densities $n=5.0 \times 10^{11} \mathrm{~cm}^{-2}$ (cf. top left panel). In this regime, $\tau_{1}(\varepsilon)$ is independent of $\varepsilon-\varepsilon_{F}$ and the relative PA and DA contribution can be tuned by changing solely the density $n$. At intermediate temperatures, the relaxation shows a crossover from the $T$-independent to the linear- $T$ regime. The total rate depending on $n$ and $T$ can be dominated either by PA 

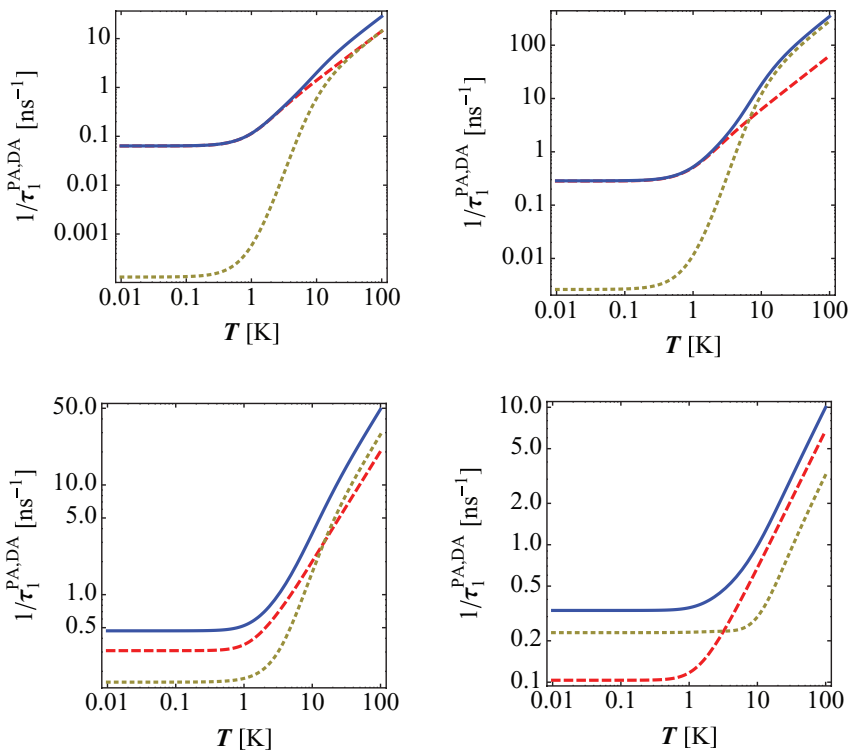

FIG. 1. (Color online) The electron momentum relaxation rate $1 / \tau_{1}^{s}\left(\varepsilon_{k}\right)$ as a function of $T$ for different values of the energy and density. The dashed and dotted lines correspond to the extrinsic PA and intrinsic DA phonon scattering mechanisms. The solid curve is the total rate. Four different situations are presented: (top, left) $\varepsilon_{k}-\varepsilon_{F}=3 \mathrm{~K}$ and $n=0.5 \times 10^{12} \mathrm{~cm}^{-2}$, (top, right) $\varepsilon_{k}-\varepsilon_{F}=3 \mathrm{~K}$ and $n=10 \times$ $10^{12} \mathrm{~cm}^{-2}$, (bottom, left) $\varepsilon_{k}-\varepsilon_{F}=15 \mathrm{~K}$ and $n=1.0 \times 10^{12} \mathrm{~cm}^{-2}$, and (bottom, right) $\varepsilon_{k}-\varepsilon_{F}=30 \mathrm{~K}$ and $n=0.1 \times 10^{12} \mathrm{~cm}^{-2}$.

phonon scattering with the $T^{3}$ behavior for smaller values of $n$ or by DA phonon scattering with the stronger $T^{4}$ dependence for higher values of $n$ (cf. top right panel).

In Fig. 2, we plot the exponents $\alpha(n, T)$ and $\beta(n, T)$ that describe the mobility $\mu_{s} \propto T^{-\alpha(n, T)}$ and $\mu_{s} \propto n^{\beta(n, T)}$. It is seen that the temperature and density behavior of the mobility calculated numerically for PA and DA scattering is in agreement with the above analytical findings. For any value of $n$, the $T^{-1}$ and $T^{-4}$ dependence of the mobility,
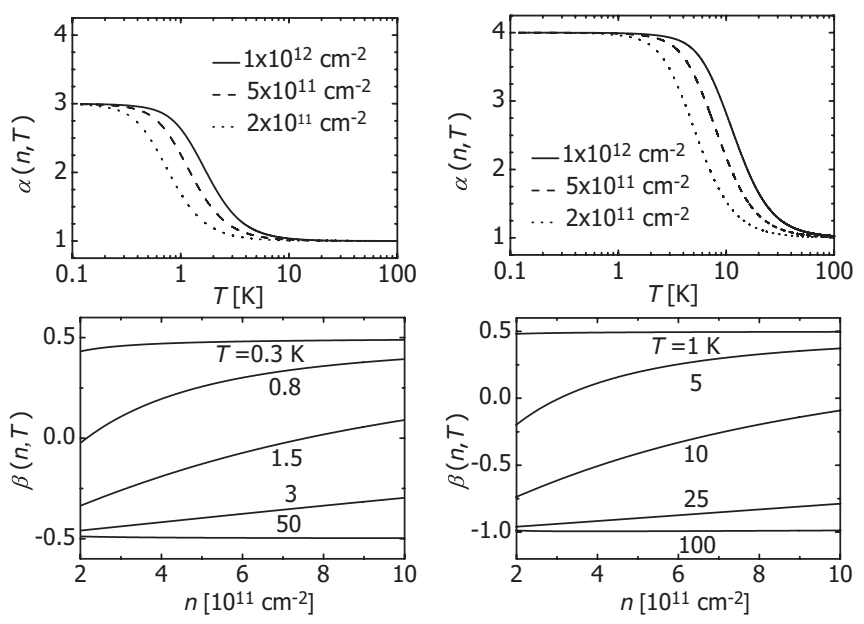

FIG. 2. The exponents $\alpha(n, T)$ (top panels) and $\beta(n, T)$ (bottom panels) describing the mobility behavior $\mu_{s} \propto T^{-\alpha(n, T)}$ and $\mu_{s} \propto$ $n^{\beta(n, T)}$ as a function of $T$ and $n$. The panels on the left- and right-hand sides depict, respectively, the PA and DA phonon limited mobilities $\mu_{\mathrm{PA}}$ and $\mu_{\mathrm{DA}}$.
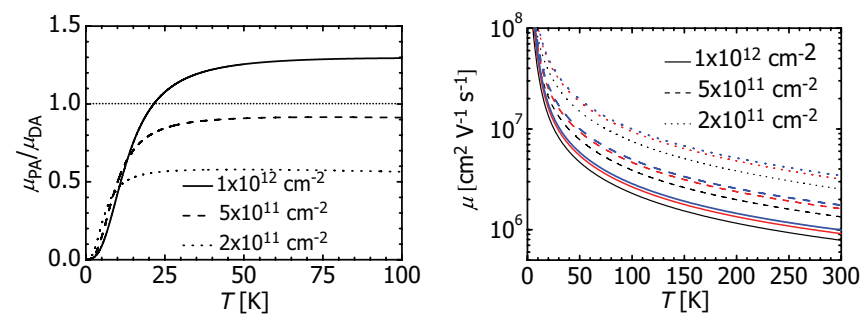

FIG. 3. (Color online) (Left) The mobility ratio $\mu_{\mathrm{PA}} / \mu_{\mathrm{DA}}$ and (right) the total mobility $\mu=\mu_{\mathrm{PA}} \mu_{\mathrm{DA}} /\left(\mu_{\mathrm{PA}}+\mu_{\mathrm{DA}}\right)$ versus $T$. The solid, dashed, and dotted curves correspond to the electron density $n=10,5$, and $2 \times 10^{11} \mathrm{~cm}^{-2}$. In the right panel, the curves in each set are calculated in three approximations for the PA phonon potential. The lower curves refer to the $\left(\hat{q}_{x} \hat{q}_{x}\right)^{2} \approx \frac{1}{4}$ and $d=0$ approximation, the middle curves take into account the angle dependence of the PA vertex with $d=0$, the upper curves include both the effect of anisotropy and of the finite distance with $d=5 \AA$.

respectively, for PA and DA phonon scattering, occurs in a wider temperature range than the $T^{-1}$ and $T^{-3}$ dependencies for DA and PA scattering. Such a reversed behavior stems from the substantial difference of the sound velocity in GaAs and graphene and can be used to distinguish between the extrinsic PA and extrinsic DA mechanisms of the momentum relaxation. As seen in the bottom panels in Fig. 2, at low $T$ the exponent $\beta(n, T) \approx \frac{1}{2}$ for both the PA and DA mechanisms. With an increase of $T$, the PA contribution to the mobility shows a crossover to the behavior with $\beta(n, T) \approx-\frac{1}{2}$, while the DA contribution to the behavior with $\beta(n, T) \approx-1$.

In Fig. 3, we show the $T$ dependence of the relative and combined contributions to the mobility made by PA and DA phonon scattering for different values of $n$. As seen in the left panel at densities $n<5 \times 10^{11} \mathrm{~cm}^{-2}$, the ratio $\mu_{\mathrm{PA}} / \mu_{\mathrm{DA}}<1$ and PA phonon scattering is the dominant mechanism, limiting the mobility both at low and high temperatures. The right panel shows the total mobility versus $T$, which we obtain applying the Matthiessen rule $1 / \mu=1 / \mu_{\mathrm{PA}}+1 / \mu_{\mathrm{DA}}$. Here, we calculate the PA contribution to the mobility including both the angle dependence of the piezoelectric potential and the finite distance $d$ between the graphene sheet and the substrate. The comparison of the upper thick curves with the lower two curves in each set of lines shows explicitly that these effects are weak.

\section{CONCLUSIONS}

In conclusion, the piezoelectric potential of acoustical phonons propagating on the surface of a GaAs substrate is an important factor limiting the mobility of Dirac fermions. At low densities, PA phonon scattering is the dominant momentum relaxation mechanism in graphene. At high temperatures, it changes qualitatively the density dependence of the mobility, while in the Bloch-Grüneisen regime it modifies the power-law dependence on temperature. Experimentally, the new power-law $T$ dependence of the relaxation rate induced by PA phonons can be measured in pure samples at low temperatures $T \lesssim T_{\mathrm{BG}}^{\mathrm{DA}}$. At higher temperatures, instead of the usual power-law $T$ dependence, experimentalists should detect the persistent linear- $T$ regime, generated by PA phonon scattering, up to temperatures much lower than the scale $T_{\mathrm{BG}}^{\mathrm{DA}}$ 
induced by DA phonons. The other important manifestation of this new PA relaxation channel, relevant to experiment, should be the predicted crossover in the density dependence of the mobility in the high- $T$ regime, which is attainable for realistic parameters in present-day graphene-GaAs heterostructures. ${ }^{33}$

\section{ACKNOWLEDGMENT}

This work was supported by the ESF-Eurocores program EuroGRAPHENE (CONGRAN project) and the Flemish Science Foundation (FWO-Vl).
*Samvel.Badalyan@ua.ac.be

${ }^{1}$ K. S. Novoselov, A. K. Geim, S. V. Morozov, D. Jiang, Y. Zhang, S. V. Dubonos, I. V. Grigorieva, and A. A. Firsov, Science 306, 666 (2004).

${ }^{2}$ A. H. C. Neto, F. Guinea, N. M. R. Peres, K. S. Novoselov, and A. K. Geim, Rev. Mod. Phys. 81, 109 (2009).

${ }^{3}$ K. S. Novoselov, A. K. Geim, S. V. Morozov, D. Jiang, Y. Zhang, S. V. Dubonos, I. V. Grigorieva, and A. A. Firsov, Nature (London) 438, 197 (2005).

${ }^{4}$ Y.-W. Tan, Y. Zhang, K. Bolotin, Y. Zhao, S. Adam, E. H. Hwang, S. Das Sarma, H. L. Stormer, and P. Kim, Phys. Rev. Lett. 99, 246803 (2007).

${ }^{5}$ A. K. Geim and A. H. MacDonald, Phys. Today 60(8), 35 (2007).

${ }^{6}$ S. Das Sarma, S. Adam, E. H. Hwang, and E. Rossi, Rev. Mod. Phys. 83, 407 (2011).

${ }^{7}$ M. I. Katsnelson and A. K. Geim, Philos. Trans. R. Soc., A 366, 195 (2008).

${ }^{8}$ Q. Li, E. H. Hwang, E. Rossi, and S. Das Sarma, Phys. Rev. Lett. 107, 156601 (2011).

${ }^{9}$ K. Kaasbjerg, K. S. Thygesen, and K. W. Jacobsen, Phys. Rev. B 85, 165440 (2012).

${ }^{10}$ K. I. Bolotin, K. J. Sikes, J. Hone, H. L. Stormer, and P. Kim, Phys. Rev. Lett. 101, 096802 (2008).

${ }^{11}$ L. A. Ponomarenko, R. Yang, T. M. Mohiuddin, M. I. Katsnelson, K. S. Novoselov, S. V. Morozov, A. A. Zhukov, F. Schedin, E. W. Hill, and A. K. Geim, Phys. Rev. Lett. 102, 206603 (2009).

${ }^{12}$ D. K. Efetov and P. Kim, Phys. Rev. Lett. 105, 256805 (2010).

${ }^{13}$ J. Yan and M. S. Fuhrer, Phys. Rev. Lett. 107, 206601 (2011).

${ }^{14}$ J. H. Chen, W. G. Cullen, C. Jang, M. S. Fuhrer, and E. D. Williams, Phys. Rev. Lett. 102, 236805 (2009).

${ }^{15}$ S. V. Morozov, K. S. Novoselov, M. I. Katsnelson, F. Schedin, D. C. Elias, J. A. Jaszczak, and A. K. Geim, Phys. Rev. Lett. 100, 016602 (2008).

${ }^{16}$ M. Ishigami, J. H. Chen, W. G. Cullen, M. S. Fuhrer, and E. D. Williams, Nano Lett. 7, 1643 (2007).

${ }^{17}$ T. Stauber, N. M. R. Peres, and F. Guinea, Phys. Rev. B 76, 205423 (2007).

${ }^{18}$ K. Nomura and A. H. MacDonald, Phys. Rev. Lett. 98, 076602 (2007); 96, 256602 (2006).
${ }^{19}$ T. Ando, J. Phys. Soc. Jpn. 75, 074716 (2006).

${ }^{20}$ E. H. Hwang, S. Adam, and S. Das Sarma, Phys. Rev. Lett. 98, 186806 (2007).

${ }^{21}$ E. H. Hwang and S. Das Sarma, Phys. Rev. B 79, 165404 (2009).

${ }^{22}$ J. H. Chen, C. Jang, S. Adam, M. S. Fuhrer, E. D. Williams, and M. Ishigami, Nat. Phys. 4, 377 (2008).

${ }^{23}$ E. H. Hwang and S. Das Sarma, Phys. Rev. B 77, 115449 (2008).

${ }^{24}$ S. Fratini and F. Guinea, Phys. Rev. B 77, 195415 (2008).

${ }^{25}$ J.-H. Chen, C. Jang, S. Xiao, M. Ishigami, and M. S. Fuhrer, Nat. Nanotechnol. 3, 206 (2008).

${ }^{26}$ K. Zou, X. Hong, D. Keefer, and J. Zhu, Phys. Rev. Lett. 105, 126601 (2010).

${ }^{27}$ H. Min, E. H. Hwang, and S. Das Sarma, Phys. Rev. B 83, 161404(R) (2011).

${ }^{28}$ S. H. Zhang and W. Xu, AIP Advances 1, 022146 (2011).

${ }^{29}$ X. Du, I. Skachko, A. Barker, and E. Y. Andrei, Nat. Nanotechnol. 3, 491 (2008).

${ }^{30}$ R. Decker, Y. Wang, V. W. Brar, W. Regan, H.-Z. Tsai, Q. Wu, W. Gannett, A. Zettl, and M. F. Crommie, Nano Lett. 11, 2291 (2011).

${ }^{31}$ C. R. Dean, A. F. Young, P. Cadden-Zimansky, L. Wang, H. Ren, K. Watanabe, T. Taniguchi, P. Kim, J. Hone, and K. L. Shepard, Nat. Phys. 7, 693 (2011).

${ }^{32}$ F. Ding, H. Ji, Y. Chen, A. Herklotz, K. Dorr, Y. Mei, A. Rastelli, and O. G. Schmidt, Nano Lett. 10, 3453 (2010).

${ }^{33}$ M. Woszczyna, M. Friedemann, M. Gtz, E. Pesel, K. Pierz, T. Weimann, and F. J. Ahlers, Appl. Phys. Lett. 100, 164106 (2012).

${ }^{34}$ S. M. Badalyan and Y. B. Levinson, Fiz. Tverd. Tela 30, 2764 (1988) [Sov. Phys. Solid State 30, 1592 (1988)]; Phys. Lett. A 140, 62 (1989); 155, 200 (1991).

${ }^{35}$ S. M. Badalyan, Fiz. Tekh. Poluprovodn. 23, 1756 (1989) [Sov. Phys.-Semicond. 3, 1087 (1989)].

${ }^{36}$ L. D. Landau and E. M. Lifshitz, Theory of Elasticity (ButterworthHeinemann, London, 1986).

${ }^{37}$ A. Knäbchen, Y. Levinson, and O. Entin-Wohlman, Phys. Rev. B 54, 10696 (1996).

${ }^{38}$ V. Gantmakher and Y. Levinson, Carrier Scattering in Metals and Semiconductors (North-Holland, Amsterdam, 1987). 